\title{
Regional imbalances in the localization of creative capital
}

\author{
Arina Suvorova ${ }^{1 *}$ \\ ${ }^{1}$ Institute of Economics of the Ural Branch of RAS, Ekaterinburg, 620014, Russian Federation
}

\begin{abstract}
In the context of the changing role of non-material factors of development, creativity becomes an obligatory feature of a competitive economic system. This study is intended to characterize the features of the localization of creative capital in Russia. During the study, the features of the placement of components that form creative capital (associated with the cultural potential of a territory, the entrepreneurial activity of people, and innovation) were analyzed. The paper uses the statistical data of Federal State Statistics Service on the processes occurring in the regions. The methodological basis of the study is a cartographic analysis, as well as an analysis of indicators that make it possible to assess the level of interterritorial inequality and the degree of concentration of resources. It was found that the localization of the elements of each of them has its own specifics: the least homogeneous is the space of implementation of innovative processes, while the difference between regions in the parameters of entrepreneurial activity is not so pronounced. This allows concluding that there is no single algorithm for the spatial location of creative capital. It has also been found that while the capital cities (Moscow and St. Petersburg) are clear centers for the localization of creative capital, the scale of other major cities in the country does not always allow them to act as points of attraction for creative resources.
\end{abstract}

Keywords: creative capital, inter-territorial inequality, spatial concentration, region.

\section{Introduction}

Currently, when the key condition for the development of economic systems is becoming the possession of intangible assets - information, knowledge, ideas, innovations, while flexibility and the ability to implement non-standard solutions turn into the most important factors for ensuring the competitiveness of any actor, the parameters of its existing creative capital become a significant characteristic of the territory [1].

It is the ideas that are the result of creative processes that provide innovative development, contribute to the determination of optimal ways to solve problems in all spheres of economic activity and represent a valuable resource of any territorial community. At the same time, the value of ideas at the stage of their emergence, their significance for the social and economic development of the territory is not obvious: they are difficult to fix, measure and manage.

${ }^{*}$ Corresponding author: suvorova.av@uiec.ru 
This fact makes creative capital an exceedingly difficult subject to study. Scientists have not yet come to a consensus about what elements the creative capital of the territory consists, what methodology can become the basis for assessing it, what policy should be implemented in the interests of its development.

This study is devoted to examining one of the aspects of this large-scale topic identifying the specifics of the spatial localization of creative capital in the Russian Federation, and its focus is on the search for similarities and differences in the features of the placement of those components that form creative capital.

\section{Literature Review}

The thesis about the close connection of the creative capital of communities with the development of the economic systems within which they operate was disclosed in detail in a whole range of studies that have established in economic science such terms as "creative economy", "creative industries", "creative class", "creative space". So, Howkins, thinking on the features of the economy of the future, singled out industries and spheres that are significant for it, referring to those, the result of the functioning of which is original developments, new technologies, intellectual property (science, art, culture, digital technologies, etc.) [2]. Landry emphasized the importance of using creative approaches in the development of places of localization of communities - cities, thereby turning creativity into an attribute of space, making it a property of the environment that has developed within the boundaries of a certain territory [3]. In Florida's studies, creativity was not just a category that allows one to take a fresh look at the peculiarities of the organization of reproduction processes in changing conditions, but an object of quantitative assessment: he proposed the concept of "Three T" - parameters that allow characterizing the level of creativity of territorial units (metropolitan areas). This concept stands for: Talent (educational and research potential of the community), Technologies (the level of innovation development), and Tolerance (the level of tolerance towards social and national minorities) [4].

Even though a number of Florida's conclusions, as well as the indicators proposed by him, have caused quite reasonable criticism in the academic community $[5,6]$, the very idea of digitalization of creative capital is of undoubted interest. Florida's approach was developed both in a significant number of academic studies [7, 8] and in comprehensive methods designed to assess the creativity of a territory. For example, the Creative Capital Index methodology was developed using the "Three T" concept, prepared by the Calvert 22 Foundation and $\mathrm{PwC}$. The developers have supplemented the parameters that allow assessing the educational and innovative potential of territorial communities, the characteristics of their openness, with some indicators that consider the degree of development of the cultural, social, business infrastructure, the peculiarities of the policies pursued by local authorities, as well as the image of a territory. The importance of assessing the cultural potential of society and guidelines for the regulatory system in identifying the prospects for the development of the creative component of the territorial system has been repeatedly drawn by specialists in economics, urban studies, and territorial management [9-11]. Although individual studies [12] do not confirm the thesis that it is the creative communities to a greater extent than other factors that determine the level of development of large-scale economic systems, the interest in their identification, measurement and identification of ways of their integration in economic processes is high not only in the USA and Western European countries (where the concept of creative capital appeared), but also in Eastern European countries, which positively perceived the idea of increasing creativity as an important condition for increasing regional competitiveness [13]. Although in some of these countries the idea of creative capital has been translated into development practices differently, 
researchers note that "the creativity term can be used for positive alternatives, not only for criticism" [14].

One of the issues that are being developed in this kind of research is the identification of the features of the localization of creative capital: the specifics of its distribution between different points in space $[15,16]$, the features of its migration between individual cities and regions [17]. Indeed, creative capital cannot be placed over the space of a country (or region) evenly: different territories "attract" the creative class (which Florida spoke about [4]) and creative industries in different ways, have different opportunities for their development. At the same time, the conditions and logic of such "attraction" are rather ambiguous. For example, some researchers note that large cities, which are traditionally perceived as the most attractive places of localization for creative professionals $[18,19]$, are inferior to mediumsized settlements in terms of their level of cultural development losing to them in the aggregate volume of creative potential resources [11]. Thus, the patterns of the spatial distribution of the creative capital of territories require further analysis, while it can be assumed that the principles of localization of its individual components (related to culture, intellectual potential, innovation sphere, urban environment, etc.) may differ quite significantly.

\section{Materials and methods}

During the study, statistical data on 85 regions of Russia were used - a country whose individual parts are very significantly different from each other both in the scale of their economic systems and in the specifics of their resources (which makes it possible to clearly demonstrate the features of spatial imbalances, indicating the groundwork for the identification of their sources). Even though the unit of spatial analysis in relation to creative capital is traditionally a city, not a region (since highly urbanized territories are the basis for creative initiatives and innovative approaches), the objectives of this study are most satisfied by a regional analysis (due to a wider set of indicators available for use at this level).

The selected research focus identified a list of parameters of particular interest. First, these are indicators of the cultural potential of a territory. They include the number of theater spectators, the number of visits to museums, and the number of users of public libraries), characterizing to a greater extent the accessibility of the cultural environment that is created within the boundaries of a territory than the cultural potential of communities permanently localized there (visitors can be representatives of other regions and countries). It should also be noted that despite the development of digitalization processes and an increase in the availability of literary publications in electronic formats, public libraries do not lose their importance for the development of the cultural potential of residents, although they partially transform their functionality, turning into leisure centers, sites for scientific and educational events, etc. Second, the parameters of the people's entrepreneurial activity (expressed by the number of small enterprises, as well as the number of individual entrepreneurs), whose significance for characterizing the creative potential of a territorial community is determined by the creativity and willingness to independently solve emerging problems that are inherent in own business. Third, the indicators of innovative activity (on which Florida made a special stake in his methodology [4]): the number of researchers (including those with an academic degree), the number of patents issued, and the volume of expenditures on R\&D. Each of the selected indicators was analyzed in both absolute and relative units, and the considered values were obtained by averaging data over a 5-year period (2015-2019). The only exception was the data on the people's entrepreneurial activity - due to the peculiarities of statistical accounting and the impossibility of obtaining continuous observations for each year, the values for these indicators were taken for only one year. 
The set of methods that formed the basis of this study is associated with the need to consider inter-territorial disparities and the peculiarities of the spatial distribution of resources: in the course of the analysis, some indicators were used that characterize the level of interregional inequality and the degree of concentration of assets in individual areas.

So, to assess the level of inequality, the Gini coefficient (1) and the Theil index (2) were used, which can be interpreted as a measure of the predictability of the result when choosing a random object (territory) from the considered set of similar objects, which allows regarding it as a characteristic of the degree of uneven distribution of assets (resources) between territories.

$$
G=1-2 \sum_{i=1}^{n} x_{i} \text { cumy }_{i}+\sum_{i=1}^{n} x_{i} y_{i}
$$

where $G$ is the Gini coefficient; $n$ is the number of territories; $x_{i}$ is the share of the i-th territory; $y_{i}$ is the share of the total volume of resources in the i-th territory; cumy $y_{i}$ is the accumulated share of the total resources in the i-th territory.

$$
T=\frac{1}{n} \sum_{i=1}^{n} \frac{x_{i}}{x_{a v}} \ln \left(\frac{x_{i}}{x_{a v}}\right),
$$

where $T$ is the Theil index; $n$ is the number of objects (territories) under consideration; $x_{i}$ is the value of the considered indicator for the i-th territory; $x_{a v}$ is the average value of the considered indicator.

In turn, to determine the degree of concentration, a rank index was used - the HallTideman index (3), which is usually used to describe the situation in the market (from the standpoint of the specifics of the competition that has developed), although it is quite applicable for a territorial analysis:

$$
H T=\frac{1}{2\left(\sum_{i=1}^{n} R_{i} y_{i}\right)-1},
$$

where $H T$ is the Hall-Tideman index; $R_{i}$ is the rank of the $\mathrm{i}$-th municipality in the considered population; $y_{i}$ is the share of the $\mathrm{i}$-th municipality in the considered population according to the analyzed indicator.

Of particular importance is also a cartographic analysis, which makes it possible to visualize the mutual disposition of territorial units that differ from each other - regions and their groups. Integral indices were calculated to generate maps that allow correlating the location of regions that differ from each other for each of the three assessed parameters. They were obtained by summing the normalized (4) values of those indicators (relative values) that characterize each of the parameters.

$$
R_{i}=\frac{\left(X_{i}-X_{\min }\right)}{\left(X_{\max }-X_{\min }\right)},
$$

where $X_{i}$ is the value of the selected indicator for the i-th region, and $X_{\max }$ and $X_{\min }$ are its maximum and minimum values, respectively.

\section{Results}

A comparison of the values of the calculated parameters of interregional inequality for different groups of indicators evidences the unequal patterns of localization of assets that differ from each other (Table 1). The lowest values of the Gini coefficient and the Theil index (which indicates a low level of inter-territorial disparities) are typical for such an indicator as "the number of users of publicly available libraries", as well as for parameters of the people's entrepreneurial activity. This result is quite natural: the development of business initiatives is not significantly dependent on the type of economic system, the presence (or absence) of large innovation, industrial complexes, or agglomerations in a territory (although the 
economic specifics of enterprises operating within the boundaries of different regions may differ quite significantly); libraries (unlike theaters and museums) do not have clear gravitation towards medium-sized and large cities and are distributed fairly evenly throughout the country.

The highest level of inter-territorial inequality was identified when analyzing the potential for Russian regions to implement innovation. This is also a fairly obvious result: research centers (uniting both the actors of creative processes and their performance, including patents) are not located in every region, most of them are localized in the central part of the country, while in Siberia and the Far East there are only separate areas that unite such institutions. The values of the indicator characterizing the internal expenditures on R\&D to an even greater extent differ from region to region - depending not only on the presence of active innovation organizations on the territory but also on their successful operation, expressed in the volume of funds that can be directed to further development, and therefore the level of territorial inequality for this indicator is very high (the values of both the Gini coefficient and the Theil index are maximum).

Table 1. Assessment of the level of inter-territorial inequality and the degree of concentration according to the parameters under consideration.

\begin{tabular}{|c|c|c|c|}
\hline Indicators & Gini coefficient & Theil index & $\begin{array}{c}\text { Hall-Tideman } \\
\text { index }\end{array}$ \\
\hline \multicolumn{4}{|c|}{ Indicators of the cultural potential of a territory } \\
\hline Number of theater spectators & 0.588 & 0.769 & 0.029 \\
\hline Number of visits to museums & 0.675 & 1.152 & 0.036 \\
\hline Number of users of public libraries & 0.395 & 0.262 & 0.019 \\
\hline \multicolumn{4}{|c|}{ Indicators of the people's entrepreneurial activity } \\
\hline Number of small businesses & 0.555 & 0.598 & 0.026 \\
\hline Number of sole traders & 0.417 & 0.301 & 0.020 \\
\hline \multicolumn{4}{|c|}{ Indicators of innovation } \\
\hline Number of researchers & 0.790 & 1.552 & 0.056 \\
\hline $\begin{array}{l}\text { Number of researchers with academic } \\
\text { degrees }\end{array}$ & 0.781 & 1.632 & 0.054 \\
\hline Patents granted & 0.698 & 1.159 & 0.039 \\
\hline Internal R\&D expenditures & 0.832 & 1.751 & 0.070 \\
\hline
\end{tabular}

Compiled according to: Federal State Statistics Service (URL: https://eng.gks.ru/)

The values of the concentration parameters of the assets under consideration largely correlate with the values of indicators of inter-territorial inequality. At the same time, despite the fact that the scale of the Hall-Tideman index for each of the analyzed indicators is closer to the minimum, and not to the maximum value (equal to 1), it is impossible to speak of a low degree of concentration: although the resources are not spatially localized at one point, judging by some parameters (primarily related to innovation), the scale of assets spatially pulling together into separate points is quite significant. For example, the number of researchers in Moscow is 3 times more than in St. Petersburg, which ranks second in terms of this indicator, and 12 times more than in the Novosibirsk Region, which ranks fifth. As noted earlier, the emergence of centers of concentration is inevitable, especially when it comes to sufficiently mobile resources (for example, highly qualified creative professionals - the creative class whose representatives are interested in a comfortable living environment, the availability of places for their activity, allowing them to get decent professional remuneration). At the same time, it is obvious that the scale of inter-territorial inequality for resources that make up the cultural potential of a territory or reflect the people's entrepreneurial activity is much less pronounced than for the elements of innovation potential. 
For the most visual representation of the level of spatial imbalances, it makes sense to turn to the maps built using the integrated data on the three parameters considered.

Thus, most of the Russian regions occupy a middle position in the rating, which can be compiled based on the values of indicators characterizing their cultural potential (Fig. 1).

Meantime, the number of outsiders (territories significantly lagging behind the leaders in terms of the value of the integral indicator) is quite large. The greatest concern is caused by the zones of their concentration (for example, the southern part of the country is clearly distinguished, its regions are provided with the required elements of the cultural infrastructure to a much lesser extent than most other Russian regions). The low positions of the Moscow Region surrounding the capital city look somewhat unexpected. This, however, can be explained by the possibility of meeting the needs of the residents to see cultural objects by visiting those located in Moscow city (which, in turn, determines a rather low rating of the capital - in terms of the ratio of the number of users of infrastructure and residents of the city, Moscow clearly loses to a few regions).

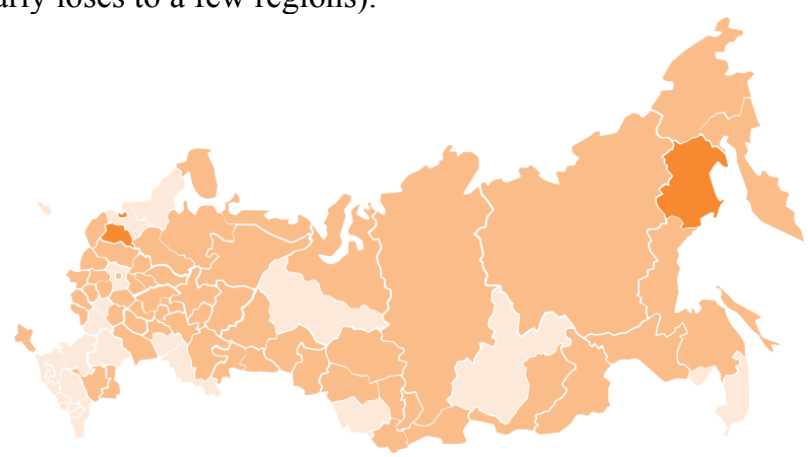

The regions with the highest indicator value are marked in a darker color

Fig. 1. Location of regions of the Russian Federation that differ in the level of cultural potential of a territory.

The scale of differences between territories differing in the parameters of entrepreneurial activity is much less significant (Fig. 2), which only confirms the conclusions drawn earlier after the analysis of absolute values.

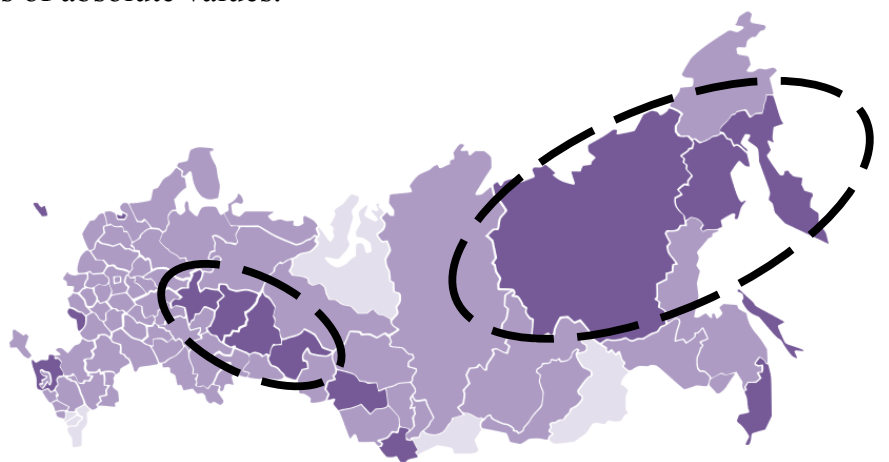

The regions with the highest indicator value are marked in a darker color

Fig. 2. The location of the regions of the Russian Federation, which differ in the parameters of the people's entrepreneurial activity.

Moreover, it is possible to identify on the map some belts that unite several neighboring top regions (schematically highlighted with dotted ovals), whose indicators of 
the development of the people's entrepreneurial initiatives exceed the parameters of neighbors (which is explained both by the specifics of the structure of the regional economy and the formation of special business support regimes). However, it should be noted that the values of the integral parameter that determined the positions of individual regions in this rating differ from each other very insignificantly (which makes it difficult to identify clear leaders or outsiders).

A completely different thing can be observed when identifying interregional differences in innovative development - as this sphere is characterized by the highest level of spatial inequality and the highest degree of concentration of assets in a limited number of areas (Fig. 3). The absolute leaders here are the cities of federal significance (Moscow and St. Petersburg), while most territories (with some exceptions) significantly lag behind them by all the considered indicators of this group.

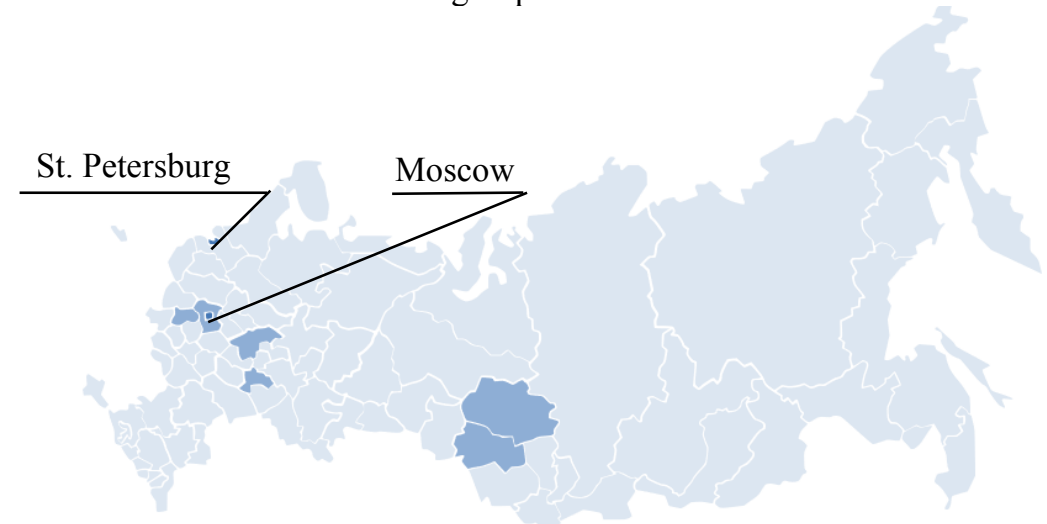

The regions with the highest indicator value are marked in a darker color

Fig. 3. Location of regions of the Russian Federation that differ in the parameters of innovation.

It can be concluded that the largest cities do accumulate a powerful innovation potential, however, not every region within which a large city is located falls into the ranks of the leaders or occupies at least a middle position in the rating. On the contrary, high values of the considered indicators in several cases are characteristic of territories that do not have large settlements (but at the same time do have advanced scientific or higher educational institutions within their borders).

\section{Discussion}

Obviously, the analysis performed touched only a small part of the comprehensive problem, which combines both the aspects of the spatial development of the country (its individual parts) and the issues of building up creative capital, which plays a special role in all economic processes occurring in today's conditions. A limited set of indicators was considered, not all methods of identifying inter-territorial disproportions have been used. Meantime, the study (despite the use of a simple toolkit) made it possible to make several interesting observations, thereby contributing to the emergence of a number of questions (so far unanswered). How appropriate is a broad approach to measuring creative capital, if its individual components obey completely different logic of "landing" at specific spatial points (and, therefore, is it necessary to separate such close categories as "creative capital", "educational capital", "human capital", and "cultural capital")? Is it possible to reduce the imbalances in the spatial localization of creative capital? How can one identify such points in the country's space 
where building up creative capital will be most effective? Finding answers to these questions can form the basis for further research on a comprehensive but important problem.

\section{Conclusion}

The study showed that individual components of creative capital demonstrated completely different patterns of their spatial localization in the country: the level of inter-territorial disparities in the location of resources of different types and the parameters of their concentration do differ. Thus, the most large-scale inter-territorial disparities are characteristic of those components of creative capital that are associated with the implementation of innovation, the most small-scale ones - with the people's entrepreneurial activity. This casts some doubt on the possibility of identifying a single pattern of the spatial location of creative capital, the zones of its gravitation (at least, within a broad interpretation of its essence, which implies taking into account the cultural and research potential of communities, the development of the social infrastructure, the openness of society and authorities, the entrepreneurial activity, etc.), and, hence, the possibility of an unambiguous answer to the question of those conditions that would allow for its growth.

The clear centers of attraction for the components of creative capital are St. Petersburg and Moscow, which at the same time occupy high positions in each of the ratings built using integral parameters (leading not only in relative but also in absolute values). Other major cities (with a population of over 1 million people) do not show a clear tendency to turn into similar centers of the second order. They are powerful magnets for creative resources at the regional level, but at the national level, other factors are gaining significance, not related (or having an indirect relationship) to the urbanistic aspects of the environment transformation (the development of the research basis, the scale of the economic system, etc.).

Acknowledgments: This work is supported by the Russian Science Foundation under grant 20-7800067.

\section{References}

1. S. Cerisola, E. Panzera, Sustainability 13(13), 7150 (2021). DOI: 10.3390/su13137150

2. J. Howkins, The creative economy: How people make money from ideas (Penguin Global, 2002)

3. C. Landry, The creative city. A toolkit for urban innovators (Routledge, 2008)

4. R. Florida, The rise of the creative class (Basic Books, 2014)

5. E.L. Glaeser, Book review of Richard Florida's "The Rise of the Creative Class" (Harvard University, 2004). URL: https://clck.ru/WAEeX

6. R. Shearmur, Work Organ. Labour Glob. 1, 31-47 (2006)

7. M. Fritsch, M. Stuetzer, Int. J. Foresight Innov. Policy 5, 7-23 (2009)

8. M. Jablonski, Cent. Eur. Manag. J. 27, 48-70 (2019)

9. D. Wolfe, M. Gertler, Innovation, creativity, and governance in Canadian city-regions, in Growing Urban Economies (2016)

10. K. Borsekova, A. Vanova, J. Surova, P. Kral, K. Tureckova, J. Nevima, S. Martinat, Land 10(3), 276 (2021)

11. V. Montalto, C. Jorge, T. Moura, S. Langedijk, M. Saisana, Cities 89, 167-185 (2019)

12. E. Tiruneh, S. Sacchetti, E. Tortia, Eur. Plan. Stud. 29(1), 75-93 (2021) 
13. K. Szara, B. Slusarczyk. Eur. Rev. 28(4), 678-692 (2020)

14. M. Czirfusz, Europa XXI 26, 85-96 (2014)

15. A. Villarreal, M. Flores, F. Sanchez, Estudios Demograficos y Urbanos 33, 149-186 (2018)

16. E. Kerimoglu, B. Karahasan, Reg. Sect. Econ. Stud. 14(1), 115-132 (2014)

17. A. Batabyal, P. Nijkamp, Int. Reg. Sci. Rev. (2021)

18. Y. Zheng, W. Xu, L. Dai, Growth Change 51(1), 464-487 (2020)

19. Y. Wei, Y. Wu, Habitat Int. 91, 102025 (2019) 